\title{
Neonatological and pulmonological management of bilateral pulmonary sequestration in a neonate
}

\author{
Andreas Woerner, Katharina Schwendener, Rainer Wolf, Mathias Nelle \\ Bern, Switzerland
}

Background: Bronchopulmonary sequestration is a lung malformation characterized by nonfunctioning lung tissue without primary communication with the tracheobronchial tree. Intrauterine complications such as mediastinal shift, pleural effusion or fetal hydrothorax can be present. We present the case of a newborn with bilateral intralobar pulmonary sequestration.

Methods: Prenatal ultrasonography in a primigravida at 20 weeks of gestation revealed echogenic masses in the right fetal hemithorax with mediastinal shift towards the left side. Serial ultrasound confirmed persistence of the lesion with otherwise appropriate fetal development. Delivery was uneventful and physical examination revealed an isolated intermittent tachypnea. Chest CT scan and CT angiography showed a bilateral intrathoracic lesion with arterial supply from the aorta. Baby lung function testing suggested possible multiple functional compartments.

Results: Right and left thoracotomy was performed at the age of 7 months. A bilateral intralobar sequestration with vascularisation from the aorta was resected. Pathological and histological examination of the resected tissue confirmed the surgical diagnosis. At the age of 24 months, the child was doing well without pulmonary complications.

Conclusions: Bilateral pulmonary sequestration requires intensive prenatal and postnatal surveillance. Though given the fact of a bilateral pulmonary sequestration, postnatal outcome showed similar favourable characteristics to an unilateral presentation. Baby lung function testing could provide additional information for optimal postnatal management and

Author Affiliations: Division of Neonatology (Woerner A, Schwendener K, Nelle M) and Division of Pediatric Radiology (Wolf R), Department of Pediatrics, University Children's Hospital, Bern, Switzerland

Corresponding Author: Andreas Woerner, Division of Neonatology, Department of Pediatrics, University Hospital of Berne, Effingerstrasse 102, CH-3010 Bern, Switzerland (Tel: +41 3163210 10; Fax: +41 3163214 05; Email: andreas.woerner@insel.ch)

C)2008, World J Pediatr. All rights reserved. timing of surgical intervention.

World J Pediatr 2008;4(4):301-304

Key words: bilateral;

bronchopulmonary sequestration;

lung function testing;

lung malformation;

neonate;

prenatal diagnosis

\section{Introduction}

$\mathrm{B}$ ronchopulmonary sequestration is a lung malformation that may be detected by antenatal ultrasonography or in the context of recurrent pulmonary infection in children. We present a very rare case of bilateral intralobar pulmonary sequestration. Given the risk of mediastinal shift, pleural effusion or fetal hydrothorax, this malformation requires intensive prenatal and postnatal surveillance with favourable outcome in this case. Lung function testing could give additional information for an optimal postnatal management of pulmonary sequestrations.

\section{Case report}

Prenatal ultrasonography in a primigravida at 20 weeks of gestation revealed echogenic masses in the right fetal hemithorax (Fig. 1) with a systemic arterial supply from the aorta (Fig. 2). A mediastinal shift towards the left side was present. Serial ultrasound examinations confirmed the persistence of the lesion, with otherwise appropriate fetal development. There was no pleural effusion or fetal hydrops. A male infant was delivered vaginally at $412 / 7$ weeks of gestation, with a birth weight of $3710 \mathrm{~g}$ and Apgar scores 8, 9 and 9 at 1, 5 and 10 minutes, respectively. Arterial and venous umbilical cord $\mathrm{pH}$ were 7.27 and 7.36, respectively. Apart from an isolated intermittent tachypnea between 60 and $90 / \mathrm{min}$, the physical examination and transcutaneous oxygen saturation were normal. On day 4, a CT scan confirmed not only a lesion in the right lower lobe measuring 55 $\times 32 \times 46 \mathrm{~mm}$ but also a distinct secondary lesion in 


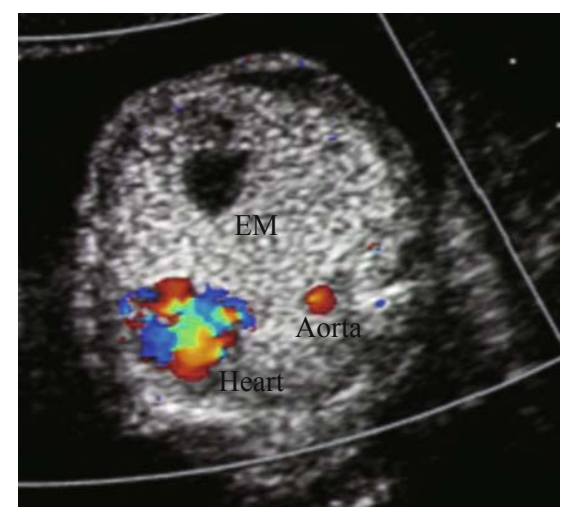

Fig. 1. Doppler ultrasonography with echogenic mass (EM) in the fetal thorax at 20 weeks of gestation.

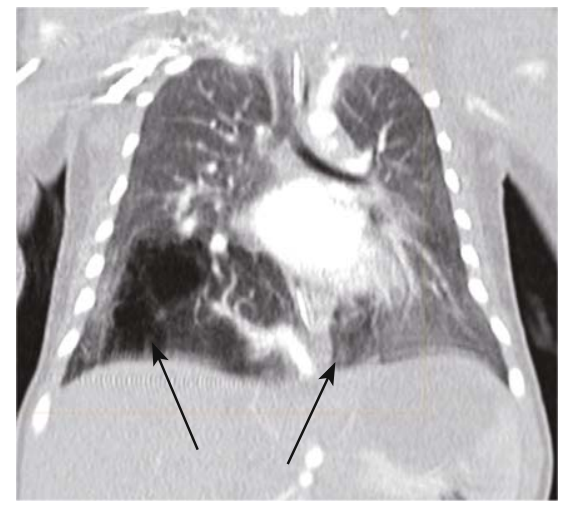

Fig. 3. Thorax CT demonstrates bilateral pulmonary sequestration in the right and left lower lobe. Figure previously published by Stern et al., J Pediatr Surg. 2007 Apr;42(4):E19-23.

the left lower lobe measuring $17 \times 26 \times 26 \mathrm{~mm}$ (Fig. 3). $2 \mathrm{D}$ reconstruction of $\mathrm{CT}$ images showed that arterial supply originated from a single celiac anomalous artery with a supradiaphragmatic bifurcation. Venous drainage from both sides appeared to drain into the left atrium (Fig. 4). Echocardiogram demonstrated normal anatomy without any signs of volume overload. On the third day of life, the patient developed clinical signs of infection which were diminished after treatment with amoxicillin and amikacin. The results of blood cultures remained negative. He was discharged home on day 10 with an intermittent tachypnea of $60-80 / \mathrm{min}$ and physical examination revealed nothing abnormal.

Further diagnostic measures included an upper gastrointestinal barium study which showed a normal presentation of the upper gastrointestinal tract without any signs for a fistula or extravasation. A baby lung function testing was done using a multibreath gas washout method. The washout pattern was compatible with significant ventilation inhomogeneities, possibly related to a lung structure with multiple functional

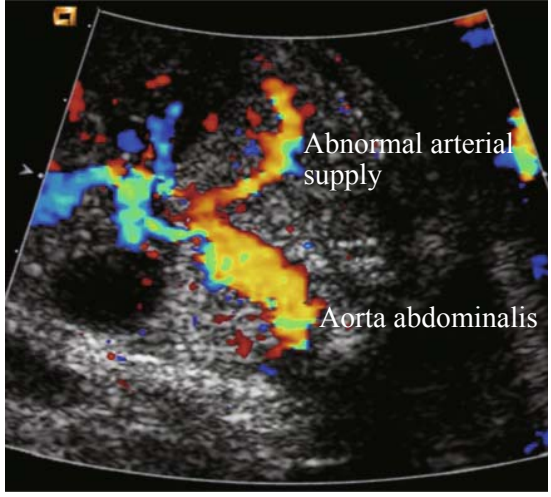

Fig. 2. Abnormal arterial supply originating from aorta abdominalis.

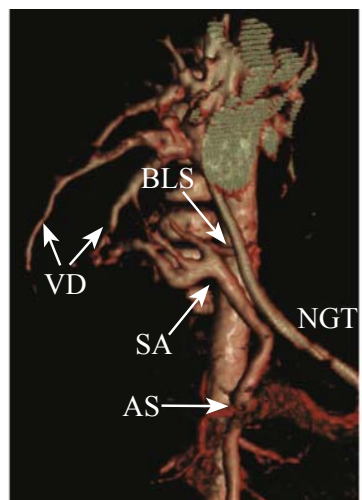

Fig. 4. 2D reconstruction of $\mathrm{CT}$ images with arterial supply (AS) originating from celiac trunk, supradiaphragmatic arborisation (SA), branch to left sided sequestration (BLS) and venous drainage (VD) to left atrium. NGT: nasogastric tube. Figure previously published by Stern et al., J Pediatr Surg. 2007 Apr;42(4):E19-23.

compartments. Chest radiography done at the age of 2, 3 and 4 months of age showed the enlargement of both sequestrations. Preoperative CT angiography confirmed the arterial supply and venous drainage as shown previously. At 7 months of age, a right and left thoracotomy revealed an intralobar sequestration on both sides with a vascularisation as described previously. On the right side, the lower lobe was resected, and on the left side, segments VII and X were resected atypically after a diagnostic thoracoscopy. Pathological and histological examination of the resected tissue confirmed the surgical diagnosis. The postoperative course was uneventful and the infant was discharged for home after 8 days. A control chest radiograph taken two weeks after the operation showed signs for atelectasis of the right middle lobe. Bronchoscopy confirmed intermittent collapse of the bronchus into the right middle lobe. An inhalation therapy with salbutamol and fluticasone was given to the patient for one month. At the age of 24 months, the child was doing well without any further pulmonary complications. 


\section{Discussion}

Pulmonary sequestration is characterized by the presence of nonfunctioning lung tissue which does not primarily communicate with the tracheobronchial tree and derives its blood supply from the aorta. It can be situated extra- or intralobarly and is usually unilateral, involving the left lower lobe in most cases. Intralobar sequestration is located within the normal lung tissue and shares the same visceral pleura. Extralobar sequestration is covered by its own pleura and is separated from the normal lung tissue. Secondary bronchial communication is more common in intra- than in extralobar sequestration, whereas communication with the gastrointestinal tract is seen more often in extralobar sequestration. ${ }^{[1]}$

Bilateral pulmonary sequestration is a rare entity, with only few cases reported in the literature. Of 15 cases of bilateral sequestration in childhood reported, 6 had intralobar presentations. ${ }^{[2-14]}$ Pulmonary sequestrations are a subgroup of bronchopulmonary foregut malformations, which also include congenital cystic adenomatoid malformation, congenital lobar emphysema and bronchogenic cyst. It is postulated that they all have a similar embryological etiology. This is also supported by the fact that in many cases mixed lesions are present. ${ }^{[15]}$ The possible mechanism, as proposed by Clements and Warner, could be a disruption of the diverticulating tracheobronchial bud's systemical vascularisation at different times of pulmonary development, resulting in different malformations. ${ }^{[16]}$

With the improvement of antenatal ultrasonographic screening, perinatal centers are confronted with even smaller thoracic cystic lesions evoking further preand postnatal management. Adzick et $\mathrm{al}^{[17]}$ reported in a series of 41 prenatally detected pulmonary sequestrations that in 28 of the 41 cases the lesions regressed or even disappeared during serial prenatal ultrasonography. The cause for lung lesion regression is unknown. However, fetuses with associated symptoms such as mediastinal shift, tension hydrothorax and nonimmune hydrops may require prenatal intervention including fetal thoracocentesis or thoracoamniotic shunt placement. The hydrops may be the result of vena cava obstruction, mediastinal shift, and cardiac compression due to thoracic mass. ${ }^{[18]}$ Tension hydrothorax appears to be the result of fluid or lymph secretion from the pulmonary sequestration. ${ }^{[19]}$ If untreated, postnatal adaptation and outcome may be complicated and even fatal in case of pulmonary hypoplasia due to impaired lung development.

In our case, the large size of pulmonary sequestration initially interpreted as an unilateral presentation and the presence of mediastinal shift to the left side required close ultrasonographic follow up. Fortunately, no tension hydrothorax or nonimmune hydrops were noted. However, the size of the lesion remained stable. The favorable postnatal adaptation, even with bilateral presentation, is in accordance with reports of other postnatally diagnosed cases. ${ }^{[20-22]}$

Baby lung function testing can provide important information to evaluate the consequences to functional pulmonary capacity. The intermittent ventilation of at least one of the sequestrations, suggested by the presence of a multi-compartment wash out curve at deep breaths, provides two informations: (1) a probably existing secondary connection of one or both sequestrations to the bronchial tree, and (2), as a consequence, an increased risk for complications due to respiratory infection, given the impaired host defense of the sequestrations. This could be helpful in further postnatal management and timing of surgical intervention.

\section{Funding: None.}

Ethical approval: Not needed.

Competing interest: None declared.

Contributors: Woerner A wrote the main body of the article under the supervision of Schwendener K and Nelle M. Wolf R provided advise on radiological aspects and $2 \mathrm{D}$ reconstruction radiological data. Nelle $\mathrm{M}$ is the guarantor.

Authors' statement: A review about this topic with emphasis on surgical management has been previously published by Stern et al., J Pediatr Surg. 2007 Apr;42(4):E19-23. Figs. 3 and 4 of this case report are published with the agreement of the author.

\section{References}

1 Carter R. Pulmonary sequestration. Ann Thorac Surg 1969;7:68-88.

2 Cerruti MM, Marmolejos F, Cacciarelli T. Bilateral intralobar pulmonary sequestration with horseshoe lung. Ann Thorac Surg 1993;55:509-510.

3 Fowler CL, Pokorny WJ, Wagner ML, Kessler MS. Review of bronchopulmonary foregut malformations. J Pediatr Surg 1988:23:793-797.

4 Gerle RD, Jaretzki A 3rd, Ashley CA, Berne AS. Congenital bronchopulmonary-foregut malformation. Pulmonary sequestration communicating with the gastrointestinal tract. N Engl J Med 1968;278:1413-1419.

5 Joy GM, Abraham MK. Bilateral communicating intralobar pulmonary sequestration. Indian Pediatr 2005;42:729-730.

6 Juettner FM, Pinter HH, Lammer G, Popper H, Friehs GB. Bilateral intralobar pulmonary sequestration: therapeutic implications. Ann Thorac Surg 1987;43:660-662.

7 Karp W. Bilateral sequestration of the lung. Am J Roentgenol 1977;128:513-515.

8 Murray ME, Given-Wilson RM, Christopher JA, Jeffrey IJ. Bilateral communicating bronchopulmonary foregut 
malformations in an infant with multiple congenital anomalies. Pediatr Radiol 1994;24:128-130.

9 Pan G, Singleton E, Nihill M, Harberg F. A case of bilateral gastric bronchopulmonary-foregut malformation. Pediatr Radiol 1989;19:463-464.

10 Pendse P, Alexander J, Khademi M, Groff DB. Pulmonary sequestration. Coexisting classic intralobar and extralobar types in a child. J Thorac Cardiovasc Surg 1972;64:127-131.

11 Roe JP, Mack JW, Shirley JH. Bilateral pulmonary sequestrations. J Thorac Cardiovasc Surg 1980;80:8-10.

12 Trudel JA, Lemire G, Rabbat AG. Bilateral sequestrations of different type with mirror-image vascularization. Chest 1977;72:256-257.

13 Wimbish KJ, Agha FP, Brady TM. Bilateral pulmonary sequestration: computed tomographic appearance. AJR Am J Roentgenol 1983;140:689-690.

14 Stern R, Berger S, Casaulta C, Raio L, Abderhalden S, Zachariou Z. Bilateral intralobar pulmonary sequestration in a newborn, case report and review of the literature on bilateral pulmonary sequestrations. J Pediatr Surg 2007;42:E19-23.

15 Conran RM, Stocker JT. Extralobar sequestration with frequently associated congenital cystic adenomatoid malformation, type 2: report of 50 cases. Pediatr Dev Pathol 1999;2:454-463.

16 Clements BS, Warner J. Pulmonary sequestration and related congenital bronchopulmonary-vascular malformations: nomenclature and classification based on anatomical and embryological considerations. Thorax 1987;42:401-408.

17 Adzick NS, Harrison MR, Crombleholme TM, Flake AW, Howell LJ. Fetal lung lesions: management and outcome. Am J Obstet Gynecol 1998;179:884-889.

18 Rice HE, Estes JM, Hedrick MH, Bealer JF, Harrison MR, Adzick NS. Congenital cystic adenomatoid malformation: a sheep model of fetal hydrops. J Pediatr Surg 1994;29: 692-696.

19 Hernanz-Schulman M, Stein SM, Neblett WW, Atkinson JB, Kirchner SG, Heller RM, et al. Pulmonary sequestration: diagnosis with color Doppler sonography and a new theory of associated hydrothorax. Radiology 1991;180:817-821.

20 Bratu I, Flageole H, Chen MF, Di Lorenzo M, Yazbeck S, Laberge JM. The multiple facets of pulmonary sequestration. J Pediatr Surg 2001;36:784-790.

21 Spinella PC, Strieper MJ, Callahan CW. Congestive heart failure in a neonate secondary to bilateral intralobar and extralobar pulmonary sequestrations. Pediatrics 1998;101(1 Pt 1):120-124.

22 Lopoo JB, Goldstein RB, Lipshutz GS, Goldberg JD, Harrison MR, Albanese CT. Fetal pulmonary sequestration: a favorable congenital lung lesion. Obstet Gynecol 1999;94:567-571.

Received July 11, 2007 Accepted after revision May 13, 2008 